\title{
Remdesivir: Critical Clinical Appraisal for COVID 19 Treatment
}

Author

Saptarshi Chatterjee

\author{
Affiliation \\ Assistant Professor, Department of Microbiology, Adamas \\ University, Kolkata \\ Key words \\ remdesivir, COVID-19, clinical studies, treatment, compas- \\ sionate use, USFDA
}

received 29.05.2020

accepted $\quad 06.10 .2020$

published online 30.10 .2020

\section{Bibliography \\ Drug Res 2021; 71: 138-148 \\ DOI $10.1055 / \mathrm{a}-1288-4078$ \\ ISSN 2194-9379}

(c) 2020. Thieme. All rights reserved.

Georg Thieme Verlag KG, Rüdigerstraße 14, 70469 Stuttgart, Germany

\section{Correspondence}

Saptarshi Chatterjee

Department of Microbiology, Adamas University,

Barasat-Barrackpore Road

Kolkata 700126

India

Tel.: + 917016636678

saptarshi.gcc09@gmail.com

\section{ABSTRACT}

Remdesivir is presently been considered as 'molecule of hope' to curb the menace of COVID19. Non-availability of any USFDA approved drug has led to several attempt of drug-repurposing and development of new therapeutic molecules. However, Remdesivir has been found to be effective against a broad range of virus including SARS, MERS and COVID 19 through in-vitro studies. Several clinical research attempt are presently being conducted showing promising result yet not conclusive. This review summarized all such clinical trials to critically appraise the usage of Remdesivir against COVID 19 along with the publications related to the results of the clinical studies. The present regulatory aspect i.e. Emergency Use Authorization (EYA) and information of molecule and plausible mechanism is also dealt.

\section{Introduction}

Since the outbreak of COVID 19, there has been extensive attempt from every sphere to come up with a suitable medicine for treatment purpose. However, till date there has been no single approved medicine for COVID 19 treatment [1] though several of them are in clinical trial and are showing promising result. Remdesivir is presently the frontrunners among the drug of choice for the treatment of COVID-19 as evidenced from clinical trials. However, there is no consolidated critical clinical summery on the activity and performance of Remdesivir in clinical settings. This article approaches a critical appreciation on the drug based on clinical evidences which is essential in present context.

\section{About the molecule}

Remdesivir is chemically known as 2-ethylbutyl (2S)-2-[[[(2R,3S, 4R,5R)-5-(4-aminopyrrolo[2,1-f][1, 2, 4]triazin-7-yl)-5-cyano-3,4dihydroxyoxolan-2-yl]methoxy-phenoxyphosphoryl]amino]propanoate (IUPAC Name) with a molecular weight of $602.6 \mathrm{~g} / \mathrm{mol}$. Remdesivir, or GS-5734 is an ATP analogue having medicinal prop- erty and used as drug [2]. The structure of Remdesivir is shown in - Fig. 1.

\section{Remdesivir as Drug}

Remdesivir (GS5734) was developed by Gilead Science, an American biopharmaceutical company as a molecule for potential treatment to Hepatitis C in 2009, but failed [3]. Since 2016 the molecule Remdesivir gained popularity as drug because of its application against Ebola virus [4]. It has also shown good efficacy against SARS CoV-2 (COVID19) through in-vitro studies [5] that has led to the popularity of the drug among medical practitioners and scientific community. Remdesivir is conserved as a broad spectrum antiviral drug. Remdesivir is a prodrug of an adenosine triphosphate (ATP) analog acting against RNA viruses [6].

\section{USFDA Approval}

The drug Remdesivir is not approved by USFDA. However, on May $1^{\text {st }} 2020$, the USFDA has provided Emergency Use Authorization (EUA) and permits the emergency use of the drug for the treatment 
of suspect and laboratory confirmed COVID 19 case in adult and children hospitalized with severe disease [7]. The EPA allows Remdesivir to be distributed and administered intravenously by health care providers, as appropriate for the treatment of COVID-19. Based on the evaluation of EUA and clinical evidence available, considering the non-availability of any drug or treatment options for COVID-19, Remdesivir is assumed to be effective. The fact sheet provides detailed guidelines in this regard. EUA is provided to Gilead Sciences Inc. that simultaneously carries out clinical trials.

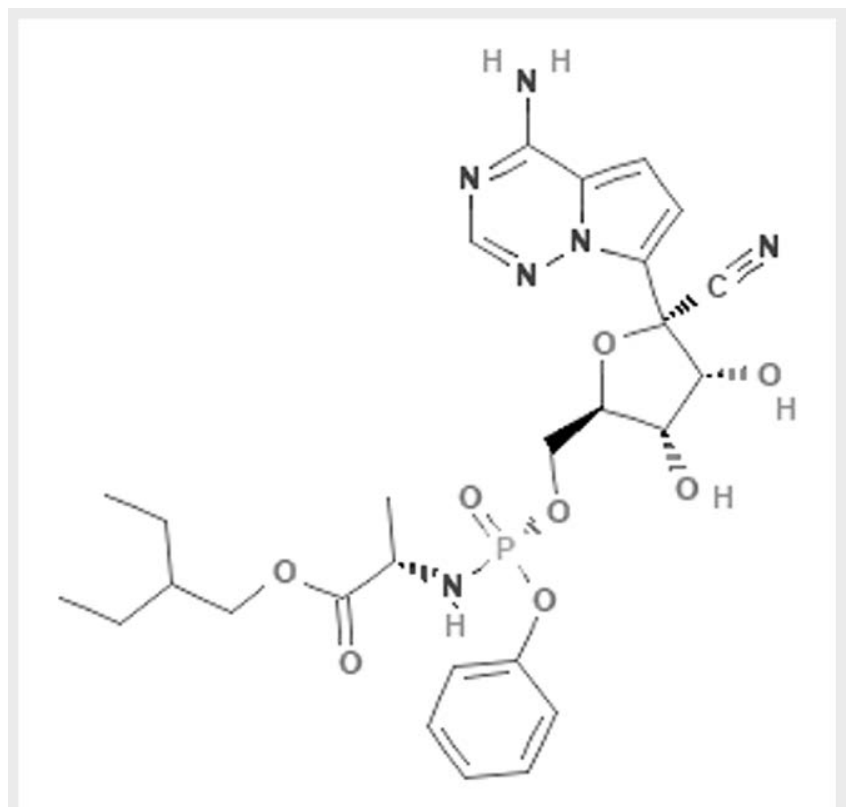

Fig. 1 Chemical Structure of Remdesivir.
EUA is issued under section 564 (FD\&C Act) was amended by the Project Bioshield Act of 2004 and was further amended by the Pandemic and All-Hazards Preparedness Reauthorization Act of 2013 (PAHPRA), the 21st Century Cures Act of 2016, and Public Law 115-92 of 2017 [8].

\section{Probable mechanism against COVID19}

Remdesivir is a prodrug of an adenosine triphosphate (ATP) analog and its triphosphate form (RDV-TP) is known to inhibit the synthesis of viral RNA by delayed chain termination method [5]. A probable molecular mechanism is deciphered that the RDT-TP binds with RNA dependent RNA Polymerase [6] and forms complex with the viral RNA and ATP to inhibit RNA synthesis [9]. - Fig 2 summarizes the plausible mechanism of Remdesivir.

\section{Dosage and use}

The USFDA through EUA suggests the use of Remdesivir, administered through intravenous (IV) infusion to treat COVID 19. Though the optimal dose and duration of treatment remains unknown, their suggestion is depicted in $>$ Table 1 [10].

\section{Other considerations and possible side effect}

It is advisable to use Remdesivir during pregnancy only if the potential benefit justifies the potential risk for the mother and the fetus. Since the pharmacokinetic analysis of Remdesivir is unavailable for patient with renal as well as hepatic imparement, its use is subject to potential risk and benefit consideration i. e. when the potential benefit outweighs the potential risk. Hepatic laboratory testing is compulsory during treatment [10].

Several infusions related reactions and symptoms including hypotension, nausea, vomiting, diaphoresis etc. are also reported in some case, that might require discontinuing the drug if clinically significant reaction takes place. Alanine aminotransferase (ALT)

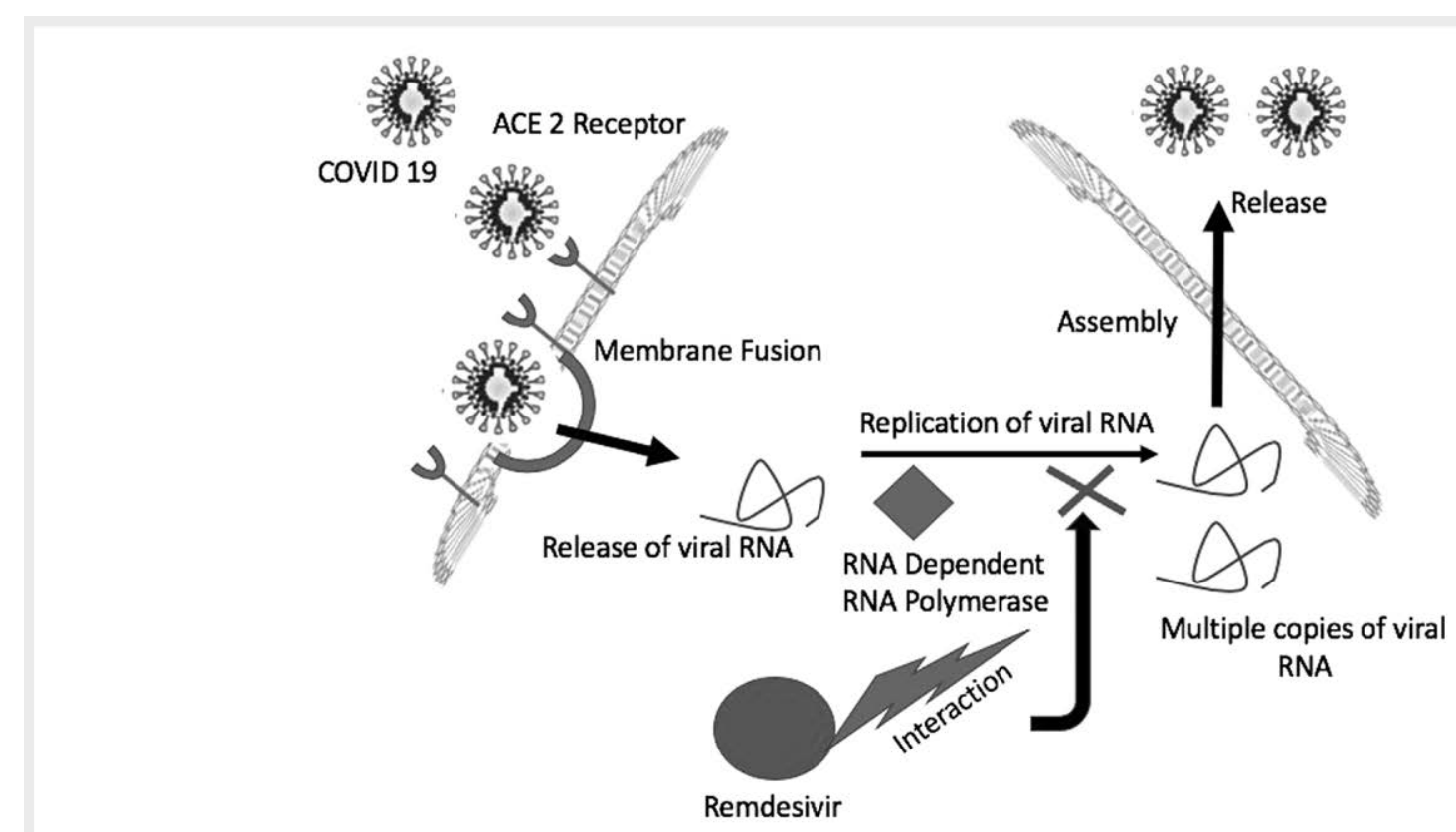

Fig. 2 Plausible mechanism of action of Remdesivir against COVID19. 
elevation is also seen [10]. The most common adverse events were increased hepatic enzymes, diarrhea, rash, renal impairment, and hypotension. In general, adverse events were more common in patients receiving invasive ventilation [11].

The empirical non-clinical data of side-effect of Remdesivir is limited to mostly in-vitro observations. However, in-vivo pharmacological studies showed that Remdesivir may transiently increase respiratory rate while no significant effect on respiration or EKG have been recorded. Remdesivir is also negative in genotoxicity studies. No renal or hepatic abnormalities were attributed to Remdesivir usage [12]. No effect on CNS and cardiovascular function have been reported [13]. Considering these facts, Remdesivir is still not beyond question [14] and remains incompletely defined in human population [15].

\section{Clinical research output}

The process of systemic review was followed ( $>$ Figure 3 ) to analyze the recent developments of Remdesivir in clinical studies from $\mathrm{NIH}$ : US National Library of Medicine [16]. At present (till Sep 2020) 30 clinical studies could be traced involving Remdesivir (Refer - Table 2) from the NIH, US National Library of Medicine Clinical Trial Registry (ClinicalTrials.gov) out of which 25 are of interventional type, 3 are observational and rest 2 are Expanded access type. Most of the clinical trials are in stage II and stage III (22 out of 30 ) indicating promising initial results yet require more time for validation and declared to be safe. Most of the trials are presently in recruiting stage while a mere 1-2 studies have been concluded. Medicinal intervention to treat COVID 19 by the process of drug repurposing as well as novel antiviral candidates could be found from

- Table 1 Dose of Remdsivir suggested by USFDA [7].

\begin{tabular}{|c|c|c|c|c|}
\hline SI No & Target Patient & Condition & Dose & Treatment Duration \\
\hline 1 & $\begin{array}{l}\text { Adults and pediatric } \\
\text { patients weigh- } \\
\text { ing } \geq 40 \mathrm{~kg}\end{array}$ & $\begin{array}{l}\text { Requiring invasive } \\
\text { mechanical ventilation } \\
\text { and/or ECMO }\end{array}$ & $\begin{array}{l}\text { Single loading dose of } 200 \mathrm{mg} \text { infused intravenously } \\
\text { over } 30-120 \text { minutes on Day } 1 \\
\text { Once-daily maintenance doses of } 100 \mathrm{mg} \text { infused } \\
\text { intravenously over } 30-120 \text { minutes for } 9 \text { days }\end{array}$ & 10 days \\
\hline 2 & $\begin{array}{l}\text { Adults and pediatric } \\
\text { patients weigh- } \\
\text { ing } \geq 40 \mathrm{~kg}\end{array}$ & $\begin{array}{l}\text { Not requiring invasive } \\
\text { mechanical ventilation } \\
\text { and/or ECMO }\end{array}$ & $\begin{array}{l}\text { Single dose of } 200 \mathrm{mg} \text { infused intravenously over } \\
30-120 \text { minutes on Day } 1 \\
\text { Once-daily maintenance doses of } 100 \mathrm{mg} \text { infused } \\
\text { intravenously over } 30-120 \text { minutes for } 4 \text { days }\end{array}$ & $\begin{array}{l}5 \text { days (May be extended } \\
\text { upto } 5 \text { additional days if } \\
\text { clinical improvement not } \\
\text { demonstrated by patient }\end{array}$ \\
\hline 3 & $\begin{array}{l}\text { Pediatric patients with } \\
\text { body weight between } \\
3.5 \mathrm{~kg} \text { and }<40 \mathrm{~kg}\end{array}$ & $\begin{array}{l}\text { Requiring invasive } \\
\text { mechanical ventilation } \\
\text { and/or ECMO }\end{array}$ & $\begin{array}{l}\text { Single loading dose of remdesivir } 5 \mathrm{mg} / \mathrm{kg} \text { IV (infused } \\
\text { over } 30-120 \mathrm{~min} \text { ) on Day } 1 \\
\text { Remdesivir } 2.5 \mathrm{mg} / \mathrm{kg} \text { IV (infused over } 30-120 \mathrm{~min} \text { ) } \\
\text { once daily for } 9 \text { days? }\end{array}$ & 10 days \\
\hline 4 & $\begin{array}{l}\text { Pediatric patients with } \\
\text { body weight between } \\
3.5 \mathrm{~kg} \text { and }<40 \mathrm{~kg}\end{array}$ & $\begin{array}{l}\text { Not requiring invasive } \\
\text { mechanical ventilation } \\
\text { and/or ECMO }\end{array}$ & $\begin{array}{l}\text { Single loading dose of remdesivir } 5 \mathrm{mg} / \mathrm{kg} \text { IV (infused } \\
\text { over } 30-120 \mathrm{~min} \text { ) on Day } 1 \\
\text { Remdesivir } 2.5 \mathrm{mg} / \mathrm{kg} \text { IV (infused over } 30-120 \mathrm{~min} \text { ) } \\
\text { once daily for } 4 \text { days }\end{array}$ & $\begin{array}{l}5 \text { days (May be extended } \\
\text { upto } 5 \text { additional days if } \\
\text { clinical improvement not } \\
\text { demonstrated by patient }\end{array}$ \\
\hline
\end{tabular}

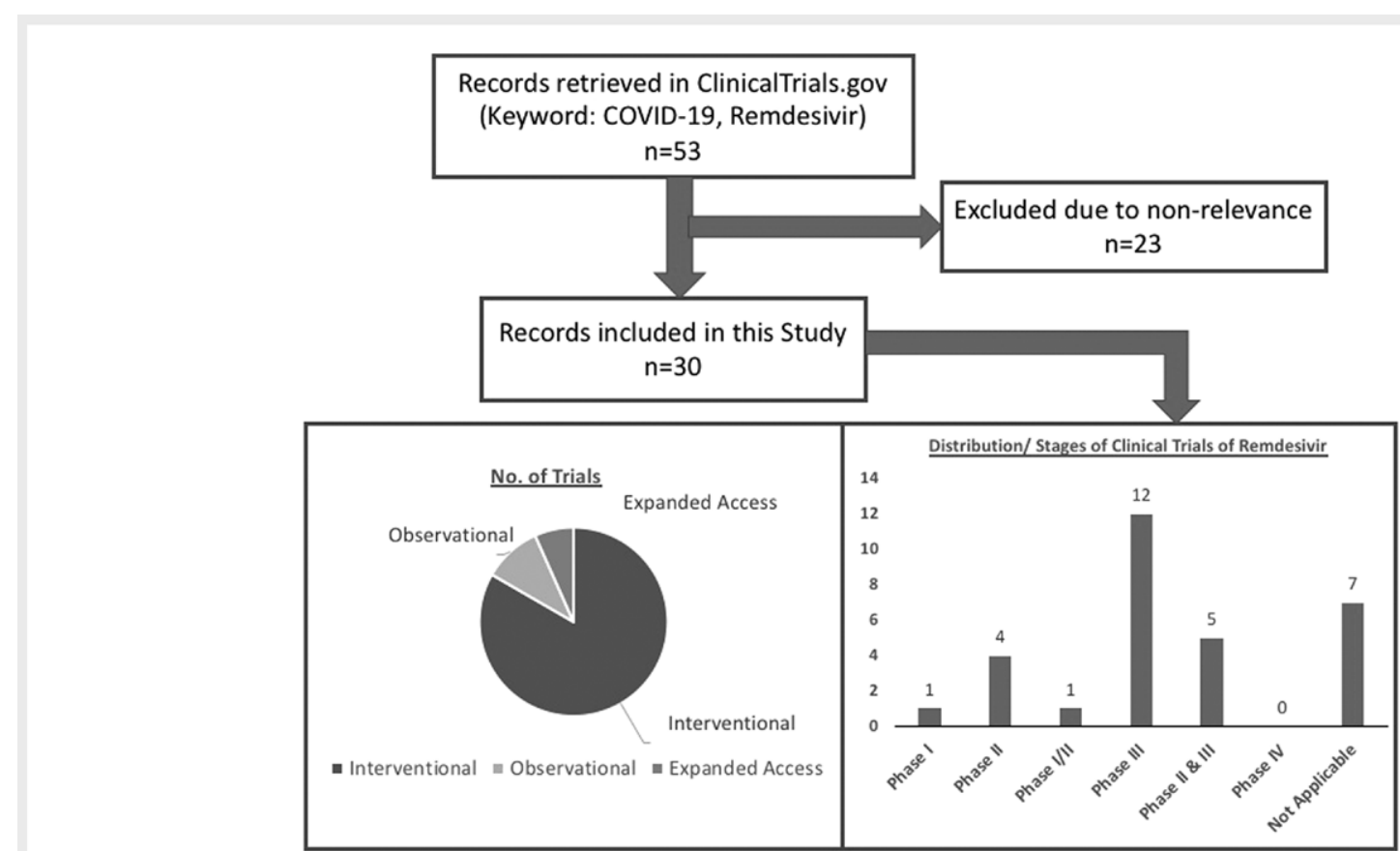

- Fig. 3 Systemic Review of Clinical Trials of remdesivir. 


\begin{tabular}{|c|c|c|c|c|c|c|}
\hline 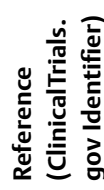 & 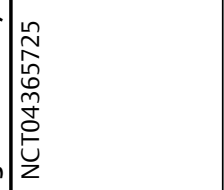 & 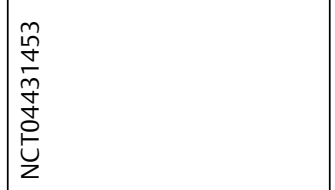 & 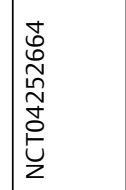 & 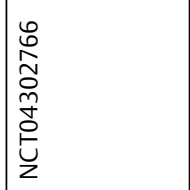 & 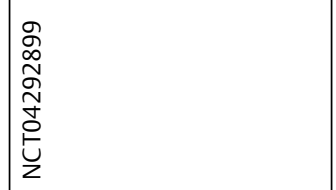 & 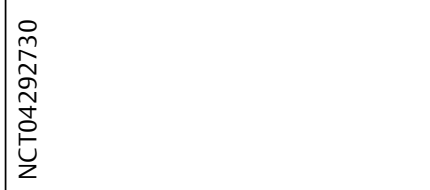 \\
\hline 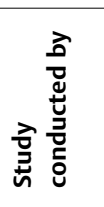 & 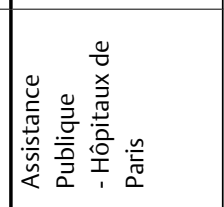 & 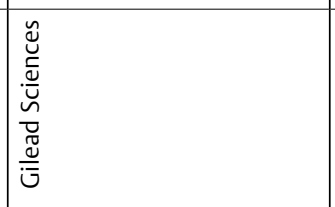 & 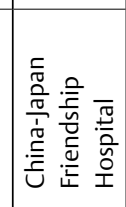 & 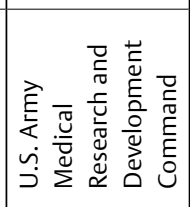 & 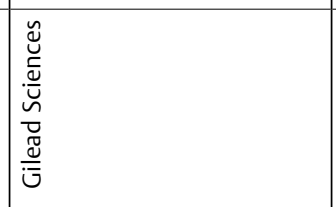 & 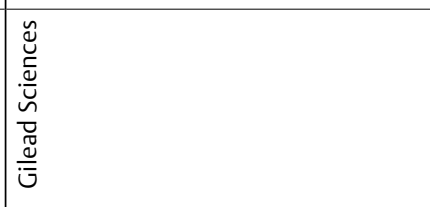 \\
\hline 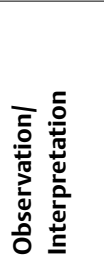 & 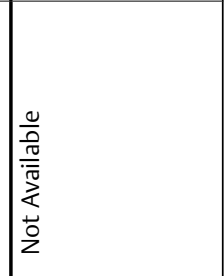 & 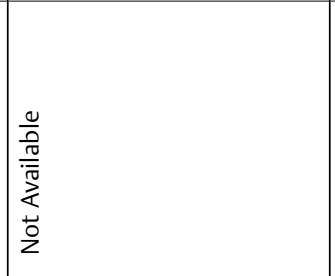 & 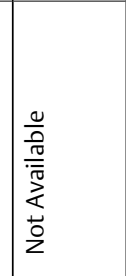 & 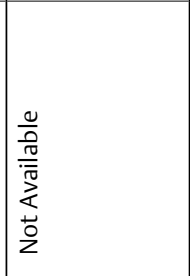 & 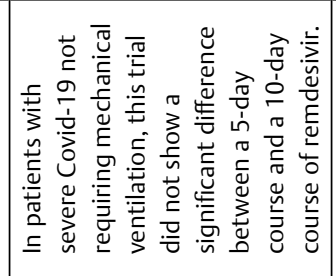 & 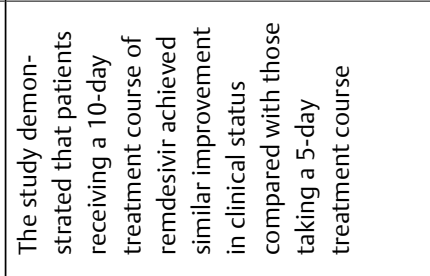 \\
\hline 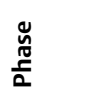 & $\frac{\pi}{z}$ & 严 & $\equiv$ & $\frac{\pi}{z}$ & $\equiv$ & $\equiv$ \\
\hline 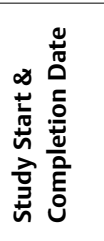 & 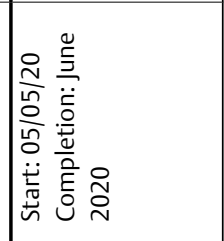 & 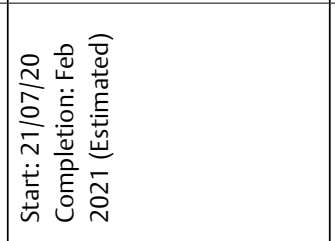 & 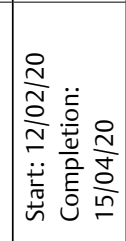 & 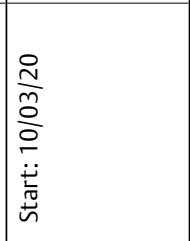 & 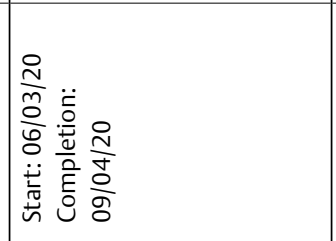 & 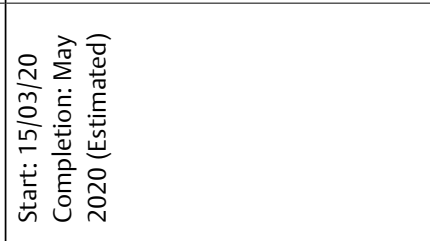 \\
\hline : & $\propto$ & $\propto$ & $\backsim$ & $<$ & u & $u$ \\
\hline 产 & 0 & - & - & 屯 & - & - \\
\hline 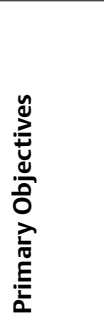 & 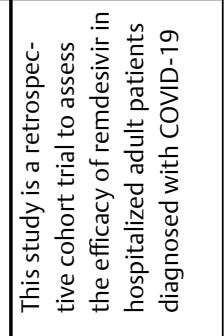 & 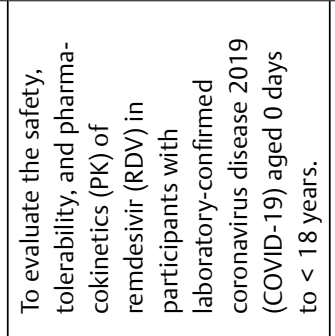 & 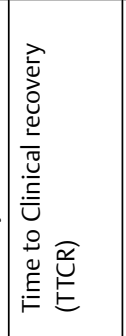 & 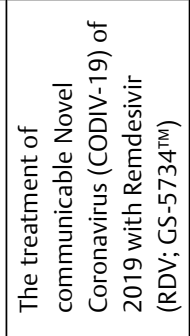 & 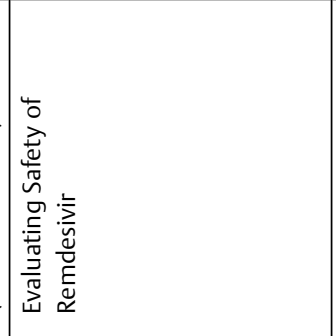 & 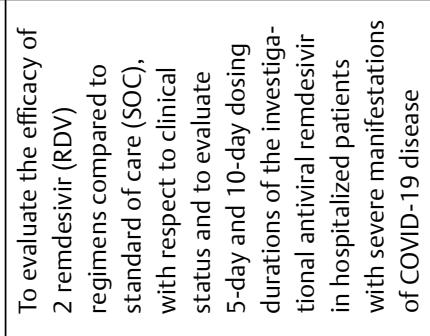 \\
\hline 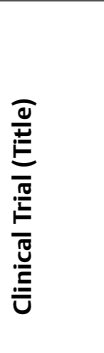 & 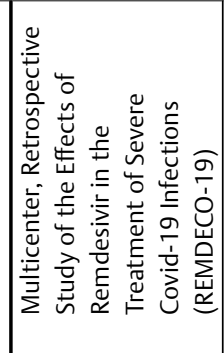 & 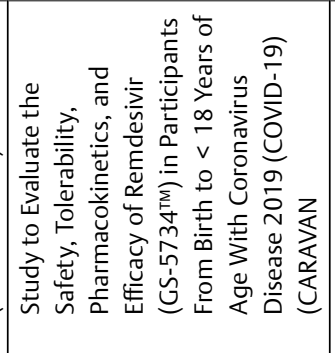 & 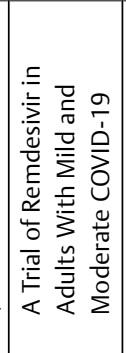 & 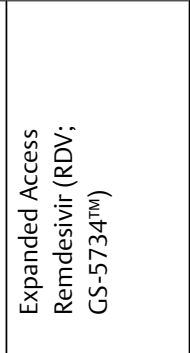 & 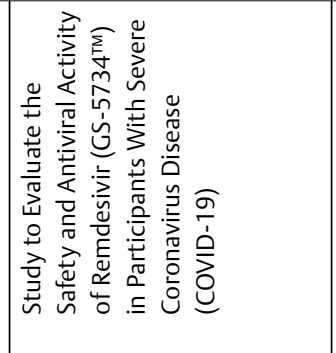 & 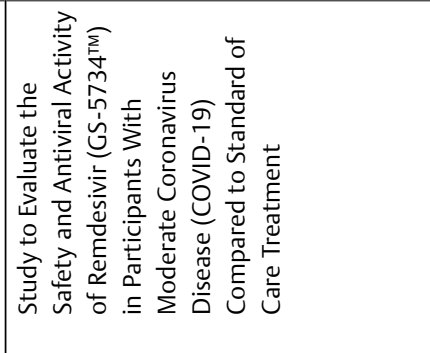 \\
\hline $\bar{n} \stackrel{0}{z}$ & - & N & m & ब & n & 0 \\
\hline
\end{tabular}




\begin{tabular}{|c|c|c|c|c|}
\hline 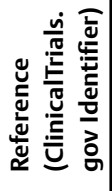 & 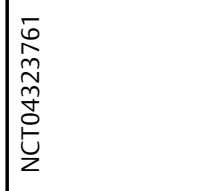 & 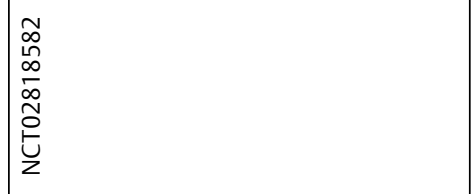 & 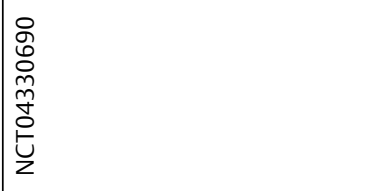 & 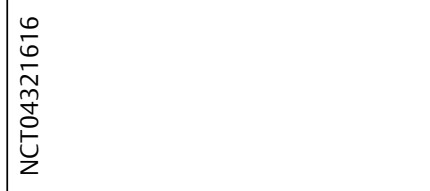 \\
\hline 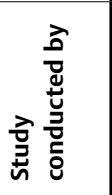 & 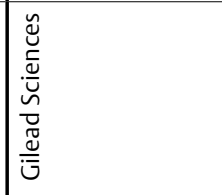 & 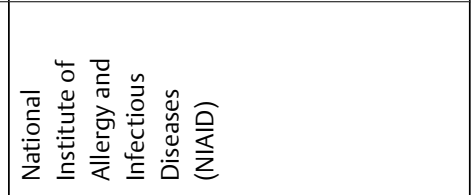 & 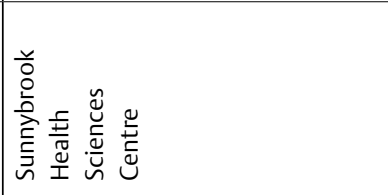 & 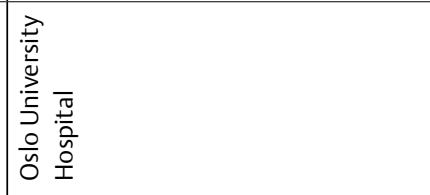 \\
\hline 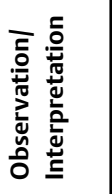 & 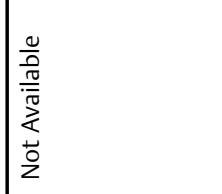 & & 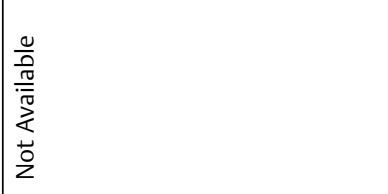 & 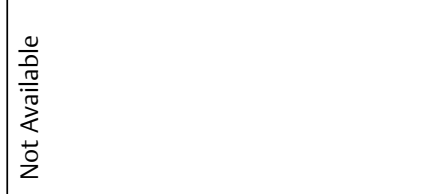 \\
\hline 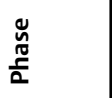 & z & $=$ & $=$ & $\begin{array}{l}\equiv \\
\stackrel{\equiv}{=}\end{array}$ \\
\hline 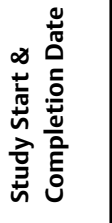 & 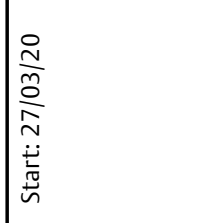 & 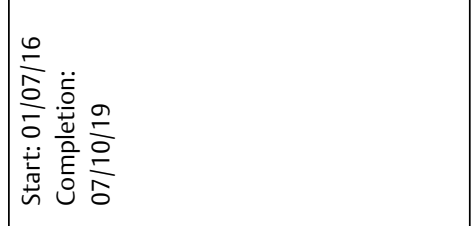 & 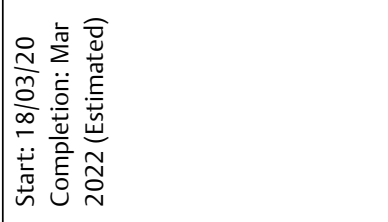 & 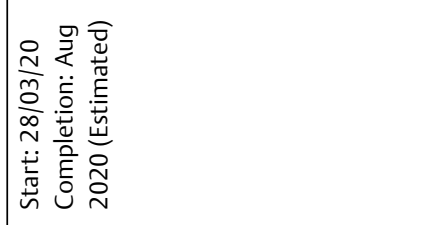 \\
\hline : & $\mid<$ & $u$ & $\propto$ & $\propto$ \\
\hline 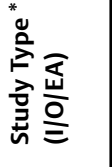 & $\varangle$ & - & - & - \\
\hline 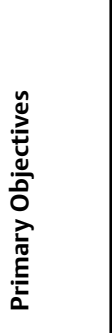 & 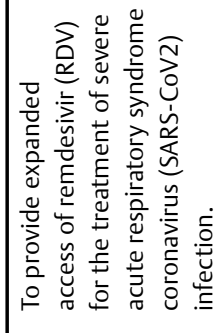 & 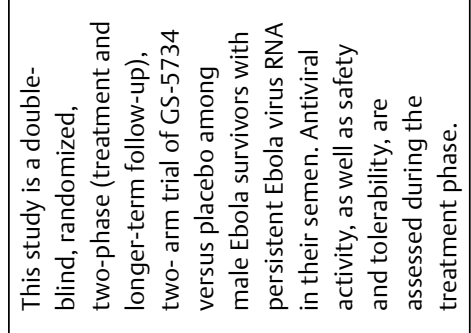 & 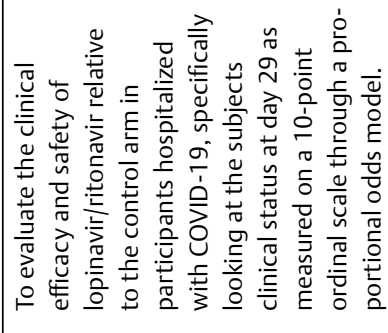 & 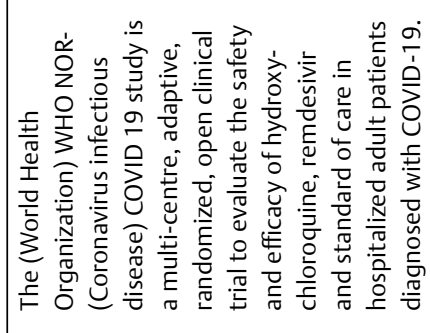 \\
\hline 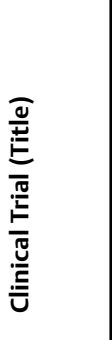 & 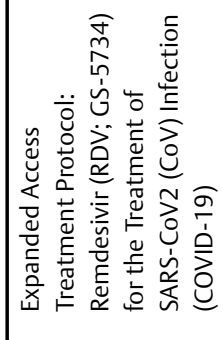 & 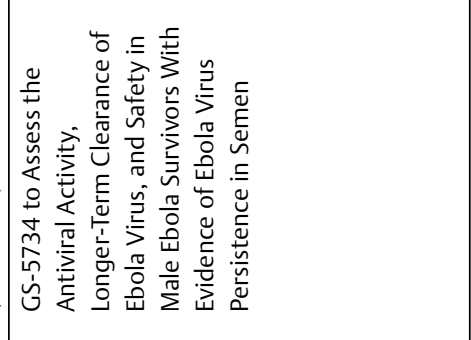 & 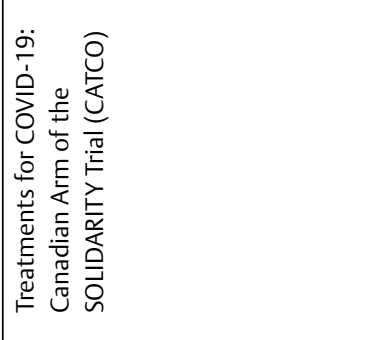 & 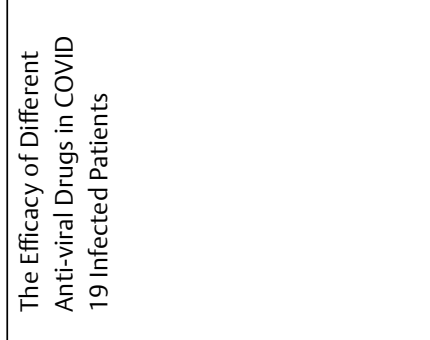 \\
\hline $\bar{n} \frac{0}{z}$ & $\wedge$ & $\infty$ & $a$ & $\circ$ \\
\hline
\end{tabular}




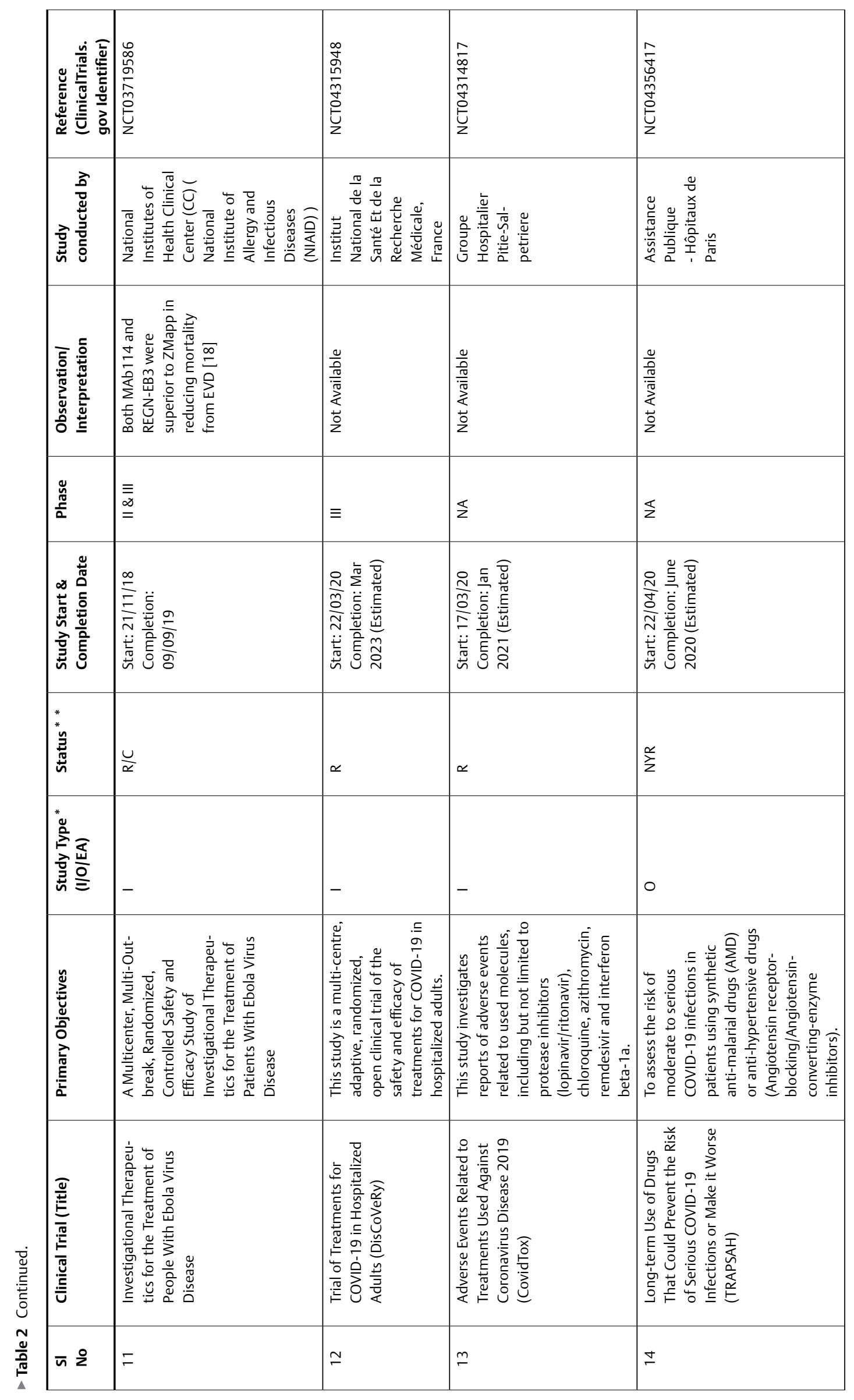




\begin{tabular}{|c|c|c|c|c|c|}
\hline 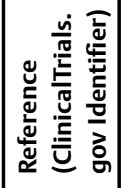 & $\begin{array}{l}0 \\
0 \\
\vdots \\
0 \\
0 \\
\tilde{0} \\
0 \\
0 \\
z\end{array}$ & 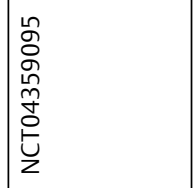 & 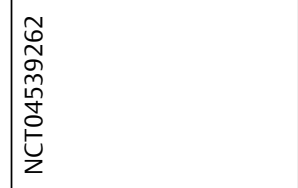 & 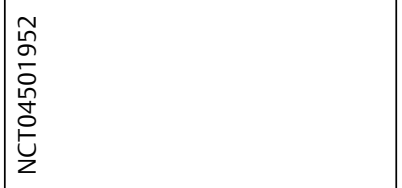 & 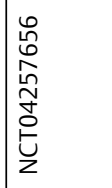 \\
\hline 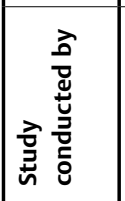 & 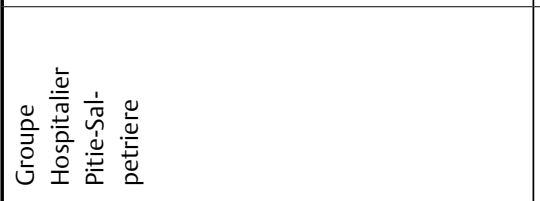 & 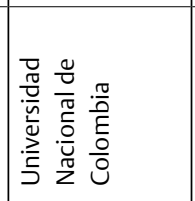 & 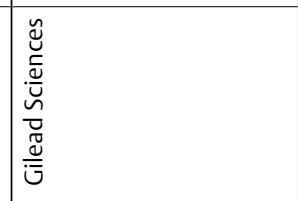 & 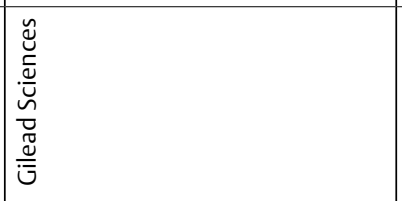 & 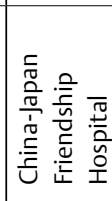 \\
\hline 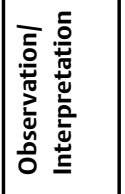 & 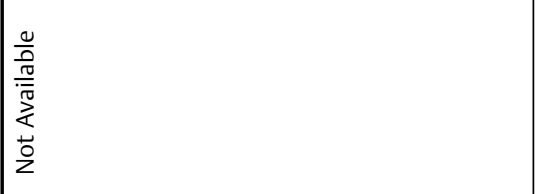 & 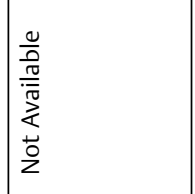 & 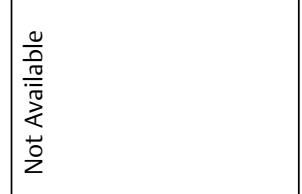 & 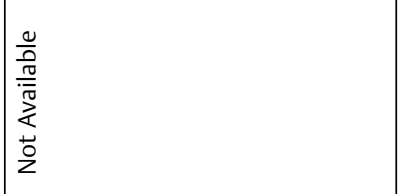 & 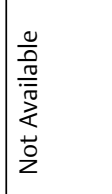 \\
\hline 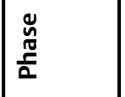 & $\Sigma$ & 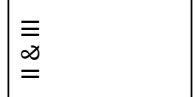 & $\equiv$ & $\equiv$ & $\equiv$ \\
\hline 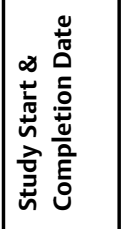 & 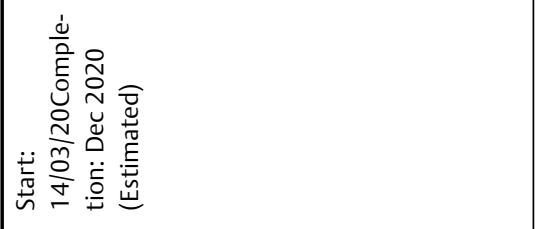 & 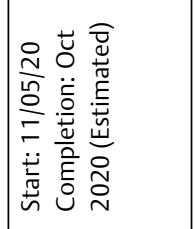 & 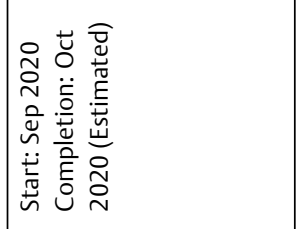 & 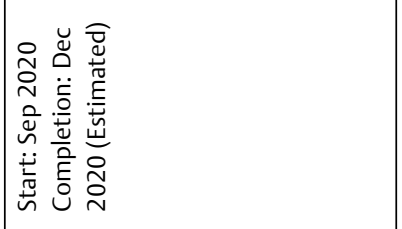 & 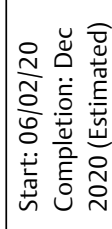 \\
\hline 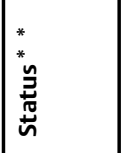 & $\propto$ & $\frac{\alpha}{\sum}$ & $\frac{\alpha}{z}$ & $\frac{\alpha}{\sum}$ & $\vdash$ \\
\hline 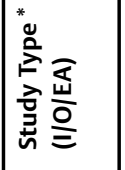 & 0 & - & - & - & - \\
\hline 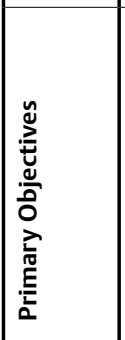 & 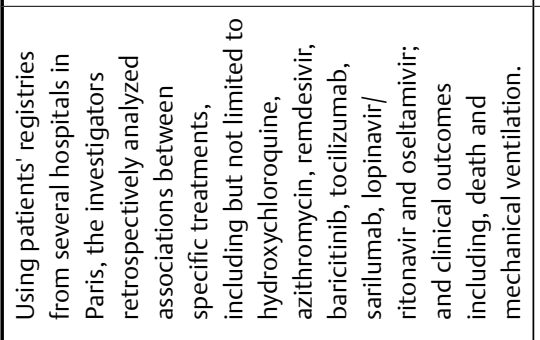 & 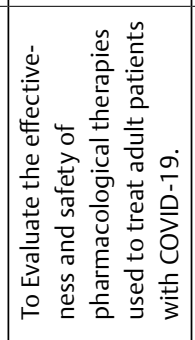 & 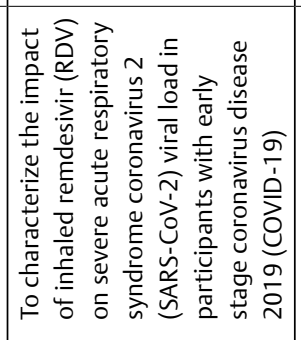 & 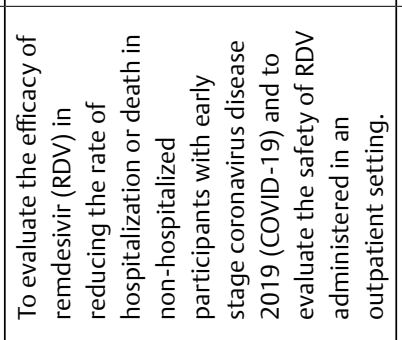 & 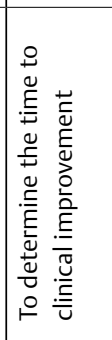 \\
\hline 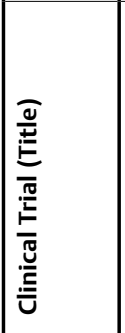 & 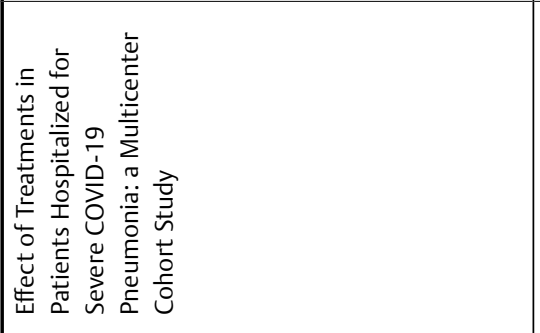 & 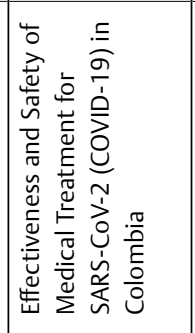 & 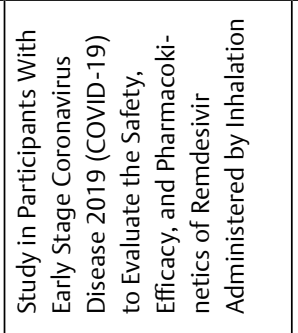 & 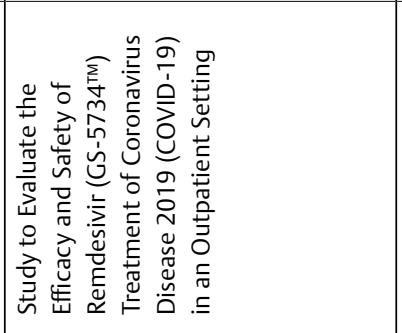 & 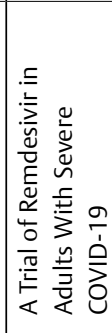 \\
\hline ज 2 & $\stackrel{n}{\llcorner}$ & $\mathscr{0}$ & $=$ & $\stackrel{\infty}{-}$ & $\stackrel{9}{\digamma}$ \\
\hline
\end{tabular}




\begin{tabular}{|c|c|c|c|c|c|c|c|}
\hline 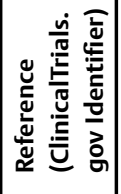 & 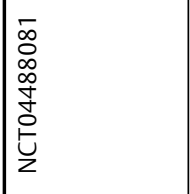 & 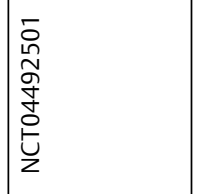 & $\begin{array}{l}\infty \\
0 \\
a \\
\vdots \\
0 \\
0 \\
\vdots \\
\vdots \\
z \\
z\end{array}$ & 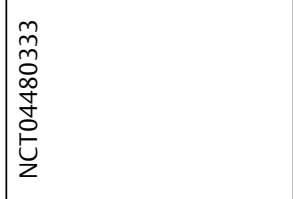 & 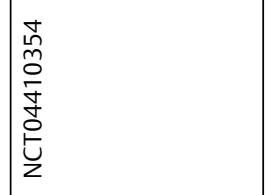 & 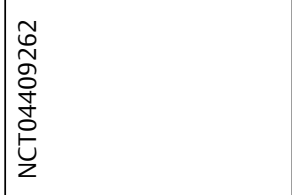 & 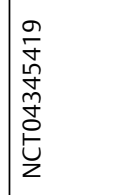 \\
\hline 咅 & 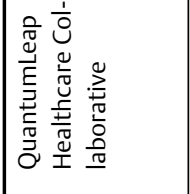 & 岀 & 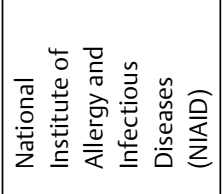 & 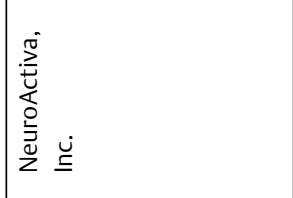 & 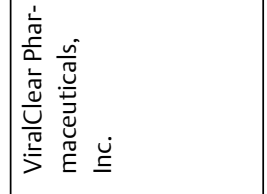 & 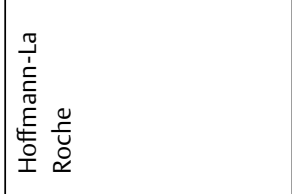 & 蒿 \\
\hline 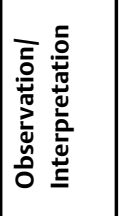 & 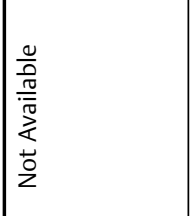 & 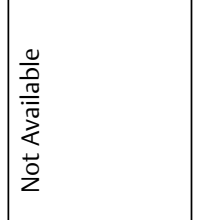 & 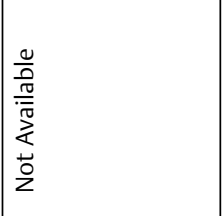 & 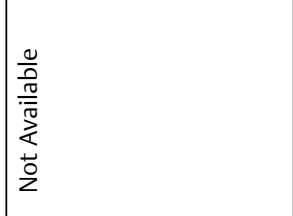 & 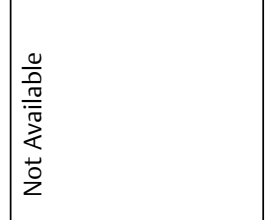 & 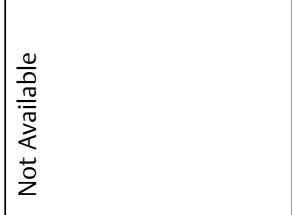 & 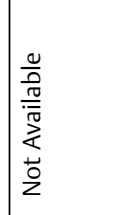 \\
\hline 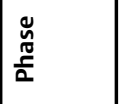 & $=$ & $\frac{\pi}{z}$ & $\equiv$ & - & $=$ & $\equiv$ & 无 \\
\hline 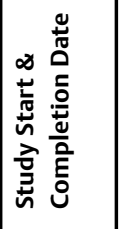 & 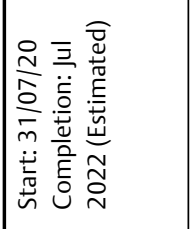 & 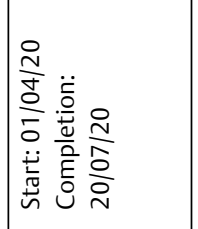 & 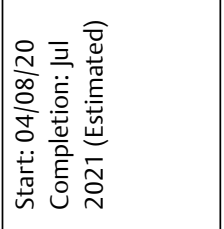 & 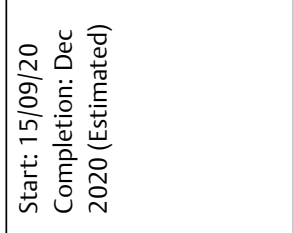 & 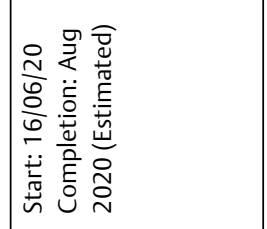 & 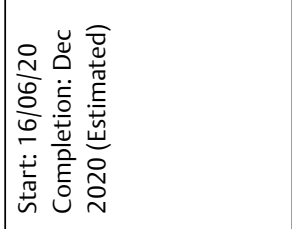 & 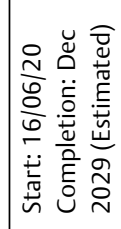 \\
\hline : & $\propto$ & $u$ & $\propto$ & $\propto$ & $\propto$ & $\propto$ & $\propto$ \\
\hline 离 & - & - & - & - & - & - & - \\
\hline 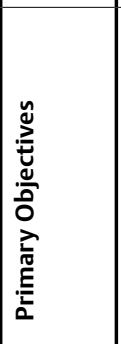 & 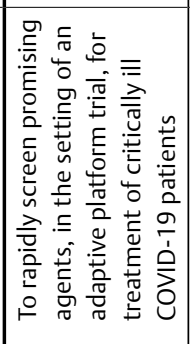 & 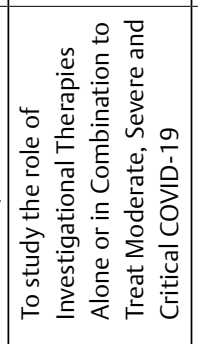 & 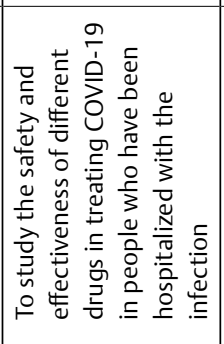 & 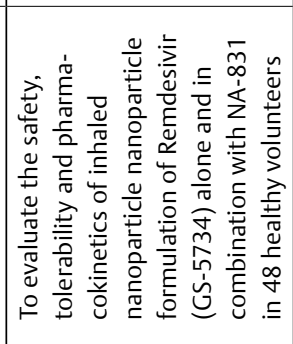 & 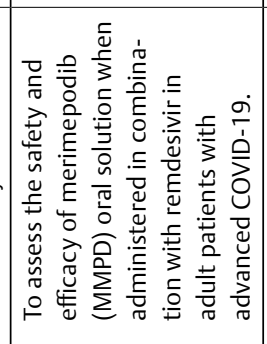 & 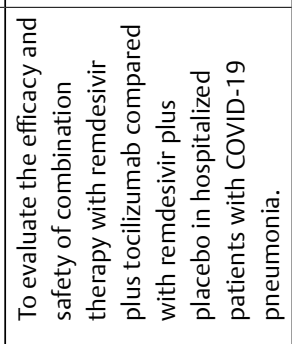 & 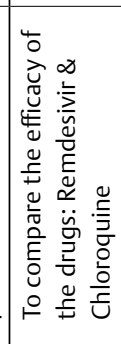 \\
\hline 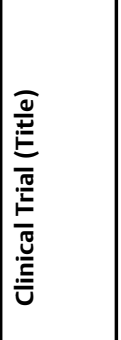 & 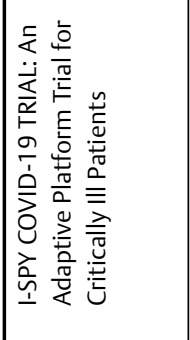 & 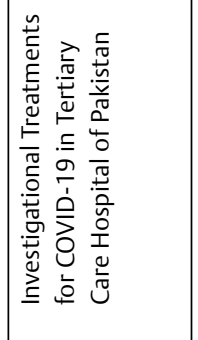 & 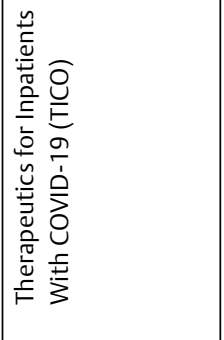 & 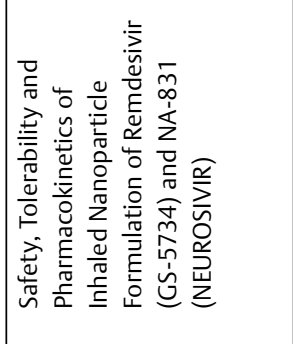 & 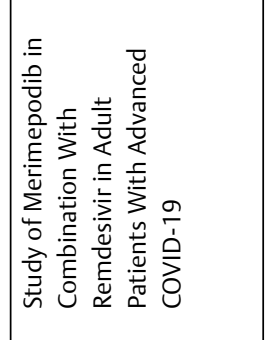 & 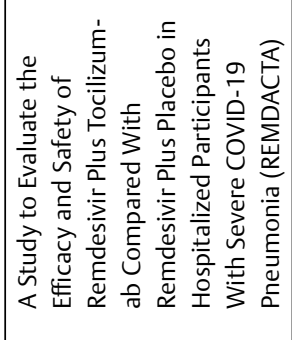 & 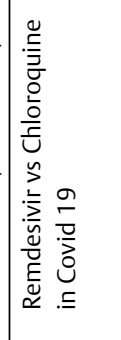 \\
\hline $\bar{n} \stackrel{2}{z}$ & is & $\bar{N}$ & N & 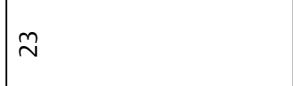 & $\stackrel{\text { I }}{ }$ & $\stackrel{4}{2}$ & $\stackrel{\sim}{\sim}$ \\
\hline
\end{tabular}




\begin{tabular}{|c|c|c|c|c|}
\hline 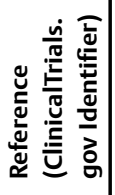 & 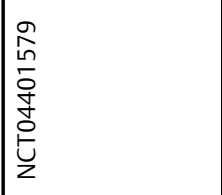 & 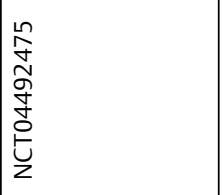 & 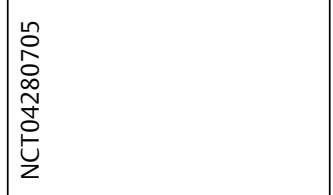 & 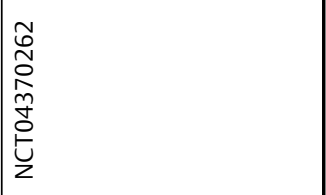 \\
\hline 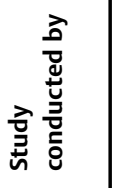 & 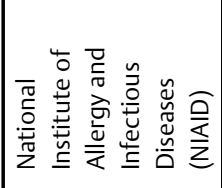 & 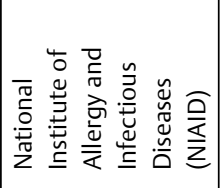 & & 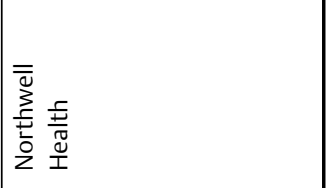 \\
\hline 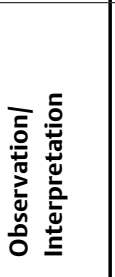 & 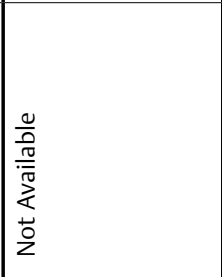 & 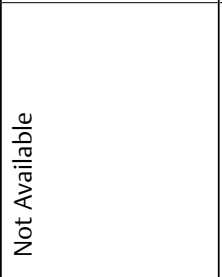 & 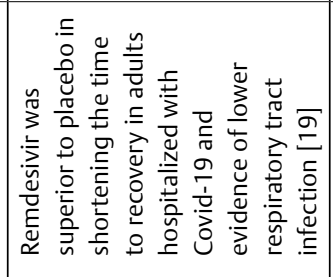 & 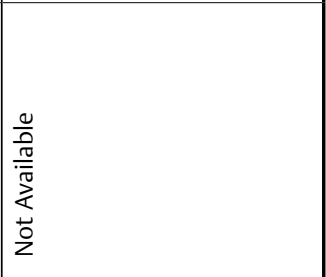 \\
\hline $\begin{array}{l}\frac{\tilde{D}}{0} \\
\frac{\tilde{c}}{a}\end{array}$ & $\equiv$ & $\equiv$ & $\equiv$ & $\equiv$ \\
\hline 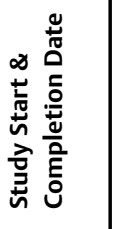 & 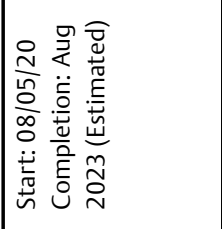 & 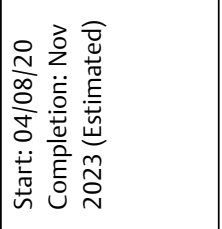 & 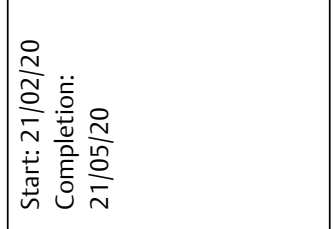 & 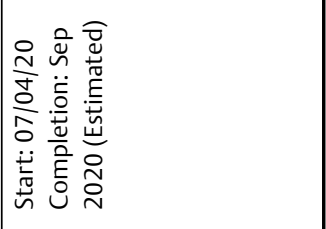 \\
\hline 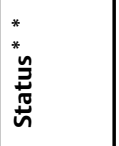 & $\frac{\tilde{z}}{z}$ & $\propto$ & $u$ & $\simeq$ \\
\hline 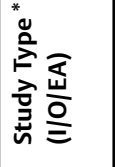 & - & - & - & - \\
\hline 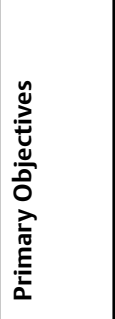 & 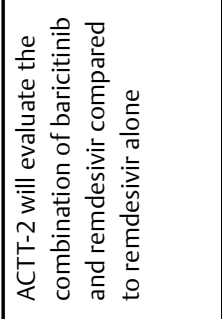 & 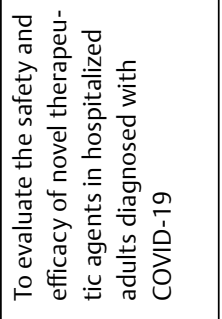 & 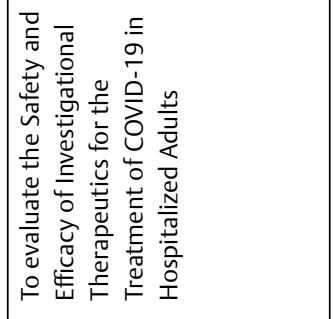 & 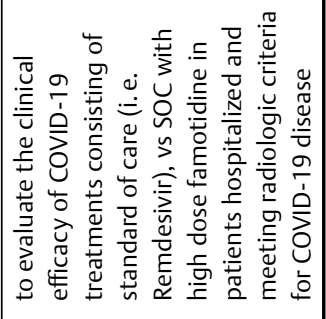 \\
\hline 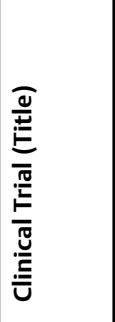 & 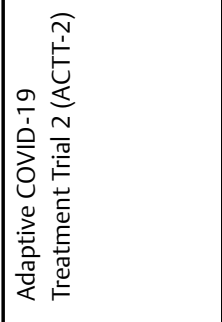 & 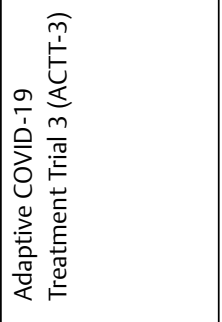 & 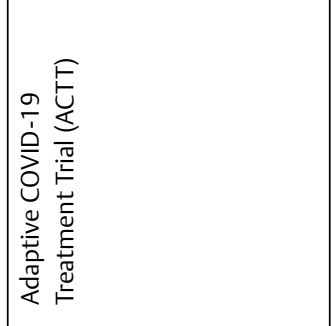 & 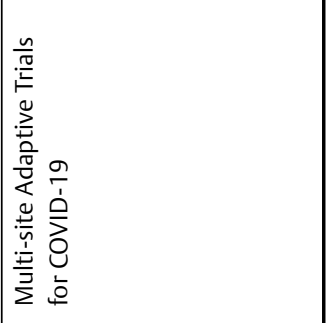 \\
\hline $\bar{n} \stackrel{0}{z}$ & 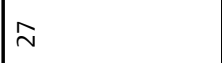 & $\stackrel{\infty}{\sim}$ & $\stackrel{\text { N }}{2}$ & $\stackrel{m}{\circ}$ \\
\hline
\end{tabular}


- Table 3 List of Publications depicting results of clinical trials on Remdesivir (Source: pubmed.gov).

\begin{tabular}{|c|c|c|c|c|}
\hline $\begin{array}{l}\text { SI } \\
\text { No }\end{array}$ & $\begin{array}{l}\text { Year of } \\
\text { Publication }\end{array}$ & Title of Publication & Significant Observation & Reference \\
\hline 1 & 2020 & $\begin{array}{l}\text { Remdesivir in adults with severe } \\
\text { COVID-19: a randomised, double- } \\
\text { blind, placebo-controlled, multicen- } \\
\text { tre trial }\end{array}$ & $\begin{array}{l}\text { This study of adult patients admitted to hospital for severe } \\
\text { COVID-19 showed thatremdesivir was not associated with } \\
\text { statistically significant clinical benefits. However, the numerical } \\
\text { reduction in time to clinical improvement in those treated earlier } \\
\text { requires confirmation in larger studies. }\end{array}$ & [17] \\
\hline 2 & 2019 & $\begin{array}{l}\text { A Randomized, Controlled Trial of } \\
\text { Ebola Virus Disease Therapeutics }\end{array}$ & $\begin{array}{l}\text { Although several experimental therapeutics for Ebola virus } \\
\text { disease (EVD) have been developed, the safety and efficacy of the } \\
\text { most promising therapies were addressed through a randomized, } \\
\text { controlled trial. A trial of } 681 \text { patients was conducted that showed } \\
\text { both MAb114 and REGN-EB3 were superior to ZMapp and } \\
\text { Remdesivir in reducing mortality from EVD. }\end{array}$ & [18] \\
\hline 3 & 2018 & $\begin{array}{l}\text { Randomised controlled trial begins } \\
\text { for Ebola therapeutics }\end{array}$ & $\begin{array}{l}\text { A trial to assess the efficacy of investigational therapeutics against } \\
\text { Ebola virus disease has been launched in DR Congo. The trial is } \\
\text { designed to test the safety, efficacy, and feasibility of investiga- } \\
\text { tional therapeutics (Remdesivir) against Ebola virus disease }\end{array}$ & {$[20]$} \\
\hline 4 & 2020 & $\begin{array}{l}\text { Compassionate remdesivir treatment } \\
\text { of severe Covid-19 pneumonia in } \\
\text { intensive care unit (ICU) and Non-ICU } \\
\text { patients: Clinical outcome and } \\
\text { differences in post-treatment } \\
\text { hospitalisation status }\end{array}$ & $\begin{array}{l}\text { This prospective (compassionate), open-label study of remdesivir, } \\
\text { which was conducted that showed remdesivir can benefit patients } \\
\text { with SARS-CoV-2 pneumonia hospitalised outside ICU where } \\
\text { clinical outcome was better and adverse events are less frequently } \\
\text { observed. }\end{array}$ & {$[21]$} \\
\hline 5 & & $\begin{array}{l}\text { Safety, Tolerability, and Pharmacoki- } \\
\text { netics of Remdesivir, An Antiviral for } \\
\text { Treatment of COVID-19, in Healthy } \\
\text { Subjects }\end{array}$ & $\begin{array}{l}\text { Remdesivir exhibited favorable safety and PK profiles that } \\
\text { supported once-daily dosing. }\end{array}$ & {$[22]$} \\
\hline 6 & 2020 & $\begin{array}{l}\text { Effect of Remdesivir vs. Standard } \\
\text { Care on Clinical Status at } 11 \text { Days in } \\
\text { Patients With Moderate COVID-19: } \\
\text { A Randomized Clinical Trial }\end{array}$ & $\begin{array}{l}\text { Randomised control trial was conducted on } 596 \text { patients with } \\
\text { moderate COVID-19. } 10 \text { day course of Remdesivir did not show } \\
\text { statistically significant difference in clinical status compared with } \\
\text { standard care while patient randomised to } 5 \text { day course showed } \\
\text { statistically significant difference in clinical status compared with } \\
\text { standard care. However the difference was uncertain clinical } \\
\text { importance. }\end{array}$ & [23] \\
\hline 7 & 2020 & $\begin{array}{l}\text { Respuestas rápidas a la pandemia de } \\
\text { COVID-19 a través de la ciencia y la } \\
\text { colaboración global: el ensayo clínico } \\
\text { Solidaridad. [Article in Spanish] }\end{array}$ & $\begin{array}{l}\text { In this trial ethical and moral obligation of patients are evaluated } \\
\text { in terms of effective treatment. The trial is a reproduction of } \\
\text { Remdesivir and other drug usage to identify whether the drugs } \\
\text { offer real time benefit to patients. }\end{array}$ & [24] \\
\hline
\end{tabular}

Feb 2020, that has peaked up since the declaration of pandemic by WHO on $12^{\text {th }}$ March 2020.

Analyzing the clinical trials from $>$ Table 2 it is evident that there is attempt to use Remdesivir alone or in combination with other drugs for the treatment of COVID 19. The non-availability of any suitable drug for the therapeutic purpose of COVID 19 as well as lack of information regarding safety issue of Remdesivir has promted its use only in hospital set-up and that too for critically ill patents. Data of adverse drug effect and long term use are expected only by the end of this year through several studies. Only a mere handful of publications are reported from the results of clinical trials $(17,18)$ while most of them are yet to be completed and still inconclusive. As a result, Remdesivir still remains as investigational drug, distant from USFDA approval.

\section{Scientific Publications}

There has been 660 Scientific publications found on the keyword 'Remdesivir; in pubmed.gov. out of which 636 are from the current year. However, a majority of them are review articles and meta- analysis while only 7 reflect results of clinical trials. The following - Table 3 summarizes the clinical results reflected in research publication.

\section{Conclusion}

Remdesivir is presently a 'molecule of hope' to the world to stop the menace of COVID19. However, it has to cross stringent safety regulations and clinical trial to be out of question. It is to be remembered that Remdesivir is still not a USFDA approved drug and its efficacy against COVID 19 are initial result that requires subsequent validation and safety/ risk analysis studies for a longer duration to act as a full proof weapon against COVID 19. Careful monitoring of patient condition and parameters are warranted during Remdesivir administration. Scientific research should also look beyond this molecule to come up with better alternatives, which remains a challenge considering present situation. 


\section{Conflict of Interest}

The author declares that there is no conflict of interest.

\section{References}

[1] National Center for Immunization and Respiratory Diseases (NCIRD), Division of Viral Diseases. Information for Clinicians on Investigational Therapeutics for Patients with COVID-19. https://www.cdc.gov/ coronavirus/2019-ncov/hcp/therapeutic-options.html

[2] Drugbank: https://www.drugbank.ca/drugs/DB14761

[3] Stephens, Bret (18 April 2020). "The Story of Remdesivir". The New York Times. p. A23. Retrieved May 2020. https://www.nytimes. com/2020/04/17/opinion/remdesivir-coronavirus.html

[4] Warren TK, Jordan R, Lo MK et al. Therapeutic efficacy of the small molecule GS-5734 against Ebola virus in rhesus monkeys. Nature 2016; 531: 381e385

[5] Gordon C], Tchesnokov EP, Woolner E et al. Remdesivir is a direct- acting antiviral that inhibits RNA-dependent RNA polymerase from severe acute respiratory syndrome coronavirus 2 with high potency. J Biol Chem 2020; https://doi.org/10.1074/jbc.RA120.013679

[6] Agostini ML, Andres EL, Sims AC et al. Coronavirus susceptibility to the antiviral remdesivir (GS-5734) is mediated by the viral polymerase and the proofreading exoribonuclease. MBio 2018; 9: e00221ee00218

[7] Coronavirus (COVID-19) Update: FDA Issues Emergency Use Authorization for Potential COVID-19 Treatment. https://www.fda.gov/ news-events/press-announcements/coronavirus-covid-19-update-fdaissues-emergency-use-authorization-potential-covid-19-treatment

[8] https://www.fda.gov/emergency-preparedness-and-response/ mcm-legal-regulatory-and-policy-framework/emergency-use-authoriz ation\#covidtherapeutics

[9] Saha A, Sharma AR, Bhattacharya M et al. Probable Molecular Mechanism of Remdesivir for the Treatment of COVID-19: Need to Know More. Archives of Medical research. 2020; https://doi. org/10.1016/j.arcmed.2020.05.001

[10] USFDA Fact Sheet For Health Care Providers: https://www.fda.gov/ media/137566/download

[11] Grein J, Ohmagari N, Shin D et al. Compassionate Use of Remdesivir for Patients with Severe Covid-19. N Engl J Med 2020; 382: 2327-2336. doi:10.1056/NEJMoa2007016

[12] https://www.ema.europa.eu/en/documents/other/summary-compassionate-use-remdesivir-gilead_en.pdf

[13] Javorac D, Grahovac L, Manić L et al. An overview of safety assessment of the medicines currently used in the treatment of COVID-19 disease [published online ahead of print, 2020 Jul 21]. Food Chem Toxicol 2020; 144: 111639. doi:10.1016/j.fct.2020.111639
[14] Chatterjee S. Status of Remdesivir: Not Yet Beyond Question!. Arch Med Res 2020; Sep 21 S0188-S4409; 30877-8. doi: 10.1016/j. arcmed.2020.09.004. Epub ahead of print. PMID: 32972773; PMCID: PMC7505045.

[15] Musa A, Pendi K, Hashemi A et al. Remdesivir for the Treatment of COVID-19: A Systematic Review of the Literature. West J Emerg Med 2020; 21: 737-741. Published 2020 May 20. doi:10.5811/westjem.2020.5.47658

[16] NIH: US National Library of Medicine https://clinicaltrials.gov/ct2/ home

[17] Wang Y, Zhang D, Du G et al. Remdesivir in adults with severe COVID-19: a randomised, double-blind, placebo-controlled, multicentre trial. Lancet 2020; May 16 395: 1569-1578. doi: 10.1016/ S0140-6736(20)31022-9. Epub 2020 Apr 29

[18] Mulangu S, Dodd LE, Davey RT Jr et al. A Randomized, Controlled Trial of Ebola Virus Disease Therapeutics. N Engl J Med 2019; Dec 12 381: 2293-2303. doi: 10.1056/NEJMoa1910993. Epub 2019 Nov 27

[19] Beigel JH, Tomashek KM, Dodd LE et al. Remdesivir for the Treatment of Covid-19 - Preliminary Report [published online ahead of print, 2020 May 22]. N Engl ] Med. 2020; NEJMoa2007764. doi:10.1056/ NEJMoa2007764

[20] Nakkazi E. Randomised controlled trial begins for Ebola therapeutics. Lancet 2018; Dec 1 392: 2338. doi: 10.1016/S0140-6736(18)33011-3 PMID: 30527603

[21] Antinori S, Cossu MV, Ridolfo AL et al. Compassionate remdesivir treatment of severe Covid-19 pneumonia in intensive care unit (ICU) and Non-ICU patients: Clinical outcome and differences in post-treatment hospitalisation status. Pharmacol Res 2020; 158: 104899 doi: 10.1016/j.phrs.2020.104899. Epub 2020 May 11. PMID: 32407959; PMCID: PMC7212963

[22] Humeniuk R, Mathias A, Cao H et al. Safety, Tolerability, and Pharmacokinetics of Remdesivir, An Antiviral for Treatment of COVID-19, in Healthy Subjects. Clin TransI Sci 2020; 13: 896-906. doi: 10.1111/cts.12840 Epub 2020 Aug 5. PMID: 32589775; PMCID: PMC7361781

[23] Spinner CD, Gottlieb RL, Criner G] et al. GS-US-540-5774 Investigators. Effect of Remdesivir vs. Standard Care on Clinical Status at 11 Days in Patients With Moderate COVID-19: A Randomized Clinical Trial. JAMA 2020; 324: 1048-1057. doi: 10.1001/jama.2020.16349. PMID: 32821939; PMCID: PMC7442954

[24] Soto A, Quiñones-Laveriano DM, Garcia P] et al. Respuestas rápidas a la pandemia de COVID-19 a través de la ciencia y la colaboración global: el ensayo clínico Solidaridad. Rev Peru Med Exp Salud Publica 2020; 37: 356-360 Spanish. doi: 10.17843/rpmesp.2020.372.5546. Epub 2020 Aug 28. PMID: 32876229 